\title{
Unsteady MHD Flow Through Porous Medium In A Rotating Parallel Plate Channel Under The Influence Of Variable Pressure Gradient
}

\author{
Shaik Sharmila ${ }^{1}$, Prof. R. Siva Prasad ${ }^{1}$ \\ ${ }^{1}$ Department of Mathematics, Sri Krishnadevaraya University, Ananthapuramu-515003, \\ Andhra Pradesh, India \\ Email : gousehalima@gmail.com
}

\begin{abstract}
In this paper, an unsteady magneto hydro dynamic (MHD) two-layered fluids flow in a horizontal channel between two parallel plates in the presence of an applied magnetic and electric field is investigated, when the whole system is rotated about an axis perpendicular to the flow. The flow is driven by a constant uniform pressure gradient in the channel bounded by two parallel insulating plates, when both fluids are considered as electrically conducting. The two fluids are assumed to be incompressible with variable properties, namely, different viscosities, thermal and electrical conductivities. Also, the transport properties of the two fluids are taken to be constant and the bounding plates are maintained at constant and equal temperatures. The governing partial differential equations are then reduced to the ordinary linear differential equations by using two-term series. Exact solutions for primary and secondary velocity distributions, also the temperatures are obtained in both fluid regions of the channel. Profiles of these solutions are plotted to discuss the effect on the flow and heat transfer characteristics, and their dependence on the governing parameters involved, such as the Hartmann number, Taylor number (rotation parameter), and ratios of the viscosities, heights, electrical and thermal conductivities. Moreover, an observation is made how the velocity and temperature distributions vary with hydro magnetic interaction in the case of steady and unsteady motions in the presence of rigid rotation.
\end{abstract}

Keywords: Porous Medium, Parallel Plate, Pressure Gradient, MHD

\section{INTRODUCTION:}

The flow of an electrically conducting fluid in the presence of a magnetic field is encountered in cosmical and geophysical fluid dynamics. The problems of fluid motion in parallel plate channels and rectangular channels have been studied by several authors due to their importance in engineering and technological fields. Subsequently, considerable attention has been also given to the study of magneto hydro dynamic flow of viscous fluids in a rotating system. New and emerging ideas have been added to the literature to possible applications in geophysics, astrophysics, engineering problems, geothermal energy, stem stimulation of oil field, food drying and heat pipes etc. The viscous fluid flow in a rotating frame of reference is of considerable importance due to the occurrence of various natural phenomena and for its application in various technological situations, which are governed by the actions of Coriolis forces.

The broad subjects of oceanography, meteorology, atmospheric science and astronomy involve some important and essential features of rotating fluids. The rotating flow of an electrically conducting fluid in the presence of a magnetic field is encountered in cosmological and geophysical fluid dynamics. Many important observations on the viscous fluid flow problems in a rotating system under different conditions and configurations have come out from the analytical studies of many investigators, namely, Greenspan and Howard [13], Holton [20], Vidyanidhi [44], Walin [45], Siegman [40], Jana and Datta [22],
Seth et al. [38], Mazumder [29], Ganapathy [9], Hayat et al. [18], Hayat and Hutter [16] and Das et al. [6]. The investigation on an oscillatory flow in a rotating channel is important from a practical point of view, because fluid oscillations may be expected in many MHD devices and natural phenomena where the fluid flow is generated due to the oscillating pressure gradient or due to vibrating plates/walls. In view of these facts, Mukherjee and Debnath [31], Seth and Jana [37], Singh [41], Ghosh [11], Ghosh and Pop [12] and Guria and Jana [14] investigated an oscillatory flow of a viscous incompressible electrically conducting fluid in a rotating channel under different conditions to analyze various aspects of the problem. Rahman and Sattar [33] studied an MHD free convection and mass transfer flow with an oscillating plate velocity and constant heat source in a rotating frame of reference.

All the above investigations have been carried out in a fluid system having single fluid flows. But many problems relating to astrophysics, geophysical fluid dynamics, aeronautics, and in petroleum industry, also in industrial applications, etc; involve multi layered-fluid flow situations. In the petroleum industry as well as in other engineering and technological fields, a stratified two-phase/two-layered fluid flow often occurs. For example, in geophysics, it is so important to study the interaction of the geomagnetic field with the hot springs/fluids in geothermal regions, in which, once the interaction of the geomagnetic field with the flow field is known, then one can easily find the temperature distribution from the well known energy equation. 


\section{Available online at www.ijrat.org}

Moreover, the temperature distribution plays an important role in MHD generators, plasma physics, turbines, etc. Also, it is a known fact that, to generate electricity, the temperature is used to run the turbine across a magnetic field. Transportation and extraction of the products of oil are other obvious applications using a two-phase system to obtain the increased flow rates in an electromagnetic pump from the possibility of reducing the power required to pump oil in a pipe line by a suitable addition of water (Shail, [39]).

There are several investigations with regards to both experimental and theoretical aspects of Magneto hydro dynamic flow problems, which are available in the literature [viz., Packham and Shail [32], Lielausis [23], Michiyoshi et al. [30], Chan [4], Chao et al. [5], Dunn [8], Gherson [10], Lohrasbi and Sahai [26], Alireza and Sahai [1], Serizawa et al. [35], Malashetty and Leela [27], Malashetty and Umavathi [28], Ramadan and Chamkha [3], Chamkha [2], Tsuyoshi Inoue and Shu-Ichiro Inutsuka [42] etc.]. Also, recent studies show that magneto hydro dynamic (MHD) flows can also be a viable option for transporting conducting fluids in micro scale systems, such as a flow inside the micro-channel networks of a lab-on-a-chip device (Haim et al., [15]; Hussameddine et al., [21]. In micro-fluidic devices, multiple fluids can be transported through a channel for different reasons. For example, an increase in mobility of a fluid may be achieved by stratification of a highly mobile fluid or mixing of two or more fluids in transit may be designed for emulsification or heat and mass transfer applications. In this regard, magnetic field-driven micro-pumps are an increasing demand due to their long-term reliability in generating flow, low power requirement and mixing efficiency (Yi et al., [47] and Weston et al., [46]).

Most of the above investigations correspond to the steady flow situations. However, a significant number of practical problems dealing with immiscible fluids are unsteady in nature. In many practical problems, it is also advantageous to consider both immiscible fluids as electrically conducting, one of which is highly electrically conducting compared to the other. The fluid of low electrical conductivity compared to the other is helpful to reduce the power required to pump the fluid in MHD pumps and flow meters. In view of these facts, Heavy and Young [19] studied oscillating two-phase channel flows. Debnath and Basu [7] discussed the unsteady slip flow in an electrically conducting two-phase fluid under transverse magnetic fields. Chamkha [3] studied the unsteady MHD convective heat and mass transfer past a semi-infinite vertical permeable moving plate with heat absorption. Umavathi et al. [43] investigated an oscillatory Hartmann two-fluid flow and heat transfer in a horizontal channel. Linga Raju and Sreedhar [25] discussed an unsteady two-fluid flow and heat transfer of conducting fluids in channels under transverse magnetic field. On the other hand, the simultaneous influence of rotation and an external magnetic field on electrically conducting two-layered/two-phase fluid systems seem to be dynamically important and physically useful etc.

\section{FORMULATION AND SOLUTION:}

Consider the unsteady MHD flow of a viscous incompressible electrically conducting fluid between two infinitely long horizontal parallel walls separated by a distance $h$. Choose a Cartesian co-ordinates system with the $x$-axis along the channel wall at $y=0$, the $y$ axis perpendicular to the channel walls and $z$-axis is normal to the $x y$-plane as shown in the figure. 1. A uniform transverse magnetic field $H_{0}$ is applied perpendicular to the channel walls. Since the channel walls are infinite in extent and the flow is unsteady, the physical variables are the function of $y$ and $t$ only.

The unsteady governing equations of motion of the flow through porous medium along $x$ and $z$-directions in a rotating frame of reference are

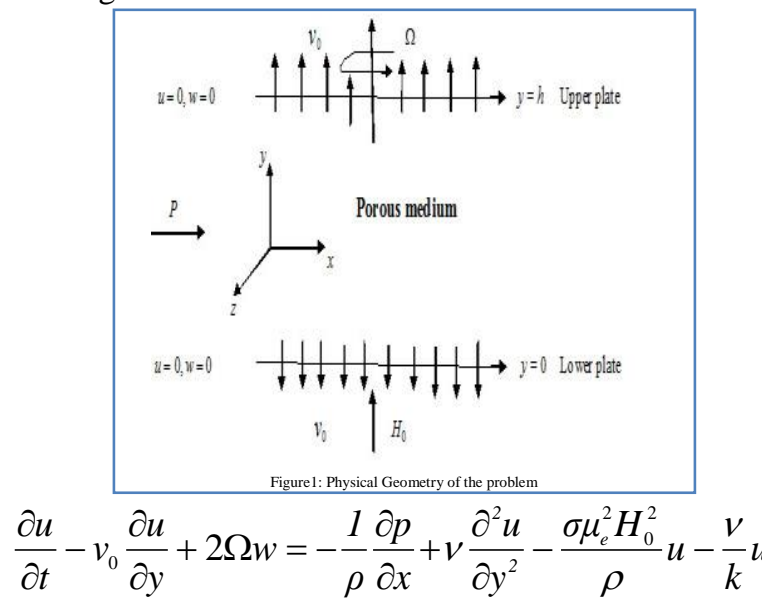

$\frac{\partial w}{\partial t}-v_{0} \frac{\partial w}{\partial y}-2 \Omega u=v \frac{\partial^{2} w}{\partial y^{2}}-\frac{\sigma \mu_{e}^{2} H_{0}^{2}}{\rho} w-\frac{v}{k} w$

where, $(u, w)$ is the velocity components along $\mathrm{O}(x, z)$ directions respectively. $\rho$ is the density of the fluid, $\mu_{e}$ is the magnetic permeability, $v$ is the coefficient of kinematic viscosity, $k$ is the permeability of the medium, $H_{0}$ is the applied magnetic field and $p$ the fluid pressure. The initial and boundary conditions are

$$
\begin{aligned}
& u=0, w=0, \quad t \leq 0, \quad 0 \leq y \leq h \\
& u=0, w=0, v=v_{0}, \quad t>0, \quad y=0 \text { and } y=h
\end{aligned}
$$

We introduce the non-dimensional variables

$x^{*}=\frac{x}{h}, y^{*}=\frac{y}{h}, u^{*}=\frac{u h}{v}, w^{*}=\frac{w h}{v}, q^{*}=\frac{q h}{v}, t^{*}=\frac{t v}{h^{2}}, \omega^{*}=\frac{\omega h^{2}}{v}, p^{*}=\frac{p h^{2}}{\rho v^{2}}$

Making use of non-dimensional variables, the equations (2.1) and (2.2) becomes to (dropping asterisks)

$$
\frac{\partial u}{\partial t}-\operatorname{Re} \frac{\partial u}{\partial y}+2 K^{2} w=f(t)+\frac{\partial^{2} u}{\partial y^{2}}-M^{2} u-D u
$$




\section{Available online at $w w w . i j r a t . o r g$}

$\frac{\partial w}{\partial t}-\operatorname{Re} \frac{\partial w}{\partial y}-2 K^{2} u=\frac{\partial^{2} w}{\partial y^{2}}-M^{2} w-D w$

Where $M^{2}=\frac{\sigma \mu_{e}^{2} H_{0}^{2} h^{2}}{\rho v}$ (Hartmann number squared) $D=\frac{k}{h^{2}}$ (Darcy parameter), $\quad K^{2}=\frac{\Omega^{2} h^{2}}{v}$ (Rotation parameter), $\operatorname{Re}=\frac{v_{0} h}{v}$ (Reynolds number) $f(t)=-\frac{1}{\rho} \frac{\partial p}{\partial x}$ (Non-dimensional pressure gradient) Corresponding non-dimensional initial and boundary conditions are

$\left.\begin{array}{ll}u=0, w=0, & t \leq 0, \quad 0 \leq y \leq 1 \\ u=0, w=0, & t>0, \quad y=0 \text { and } y=1\end{array}\right\}$

Combining equations (2.4) and (2.5), Let

$q=u+i w$ and $i=\sqrt{-1}$ we get

$\frac{\partial q}{\partial t}-\operatorname{Re} \frac{\partial q}{\partial y}=f(t)+\frac{\partial^{2} q}{\partial y^{2}}-\left(M^{2}-2 i K^{2}+D\right) q$

The initial and boundary conditions are

$q=0, \quad t \leq 0, \quad 0 \leq y \leq 1$

$q=0, \quad t>0, \quad y=0$ and $y=1$

Taking the Laplace transform of the equation (2.7), we have

$\frac{d^{2} \bar{q}}{d y^{2}}+\operatorname{Re} \frac{d \bar{q}}{d y}-\left(M^{2}-2 i K^{2}+D\right) \bar{q}=\bar{f}(s)$

where $\bar{f}(s)$ is the Laplace transform of $f(t)$,

The transformed boundary conditions are

$\bar{q}(0, s)=0$, and $\bar{q}(1, s)=0$

The solution of the equation (2.9) subjected to the boundary conditions (2.10) are

given by

$\bar{q}(y, s)=\frac{\bar{f}(s)}{\lambda_{1}+s}\left[\begin{array}{l}1-e^{-\frac{1}{2} \operatorname{Rey} y} \frac{\sinh \sqrt{\lambda_{2}+s}(1-y)}{\sinh \sqrt{\lambda_{2}+s}} \\ -e^{-\frac{1}{2} \operatorname{Re}(1-y)} \frac{\sinh \sqrt{\lambda_{2}+s}(y)}{\sinh \sqrt{\lambda_{2}+s}}\end{array}\right]$

where $\lambda_{1}=M^{2}-2 i K^{2}+D$ and

$\lambda_{2}=\frac{\mathrm{Re}^{2}}{4}+M^{2}-2 i K^{2}+D$ and we assume

$f(t)=P_{0}+P_{1} e^{i \omega t}+P_{2} e^{-i \omega t}$

where $\omega$ is the frequency of oscillations; $P_{0}, P_{1}$ and

$P_{2}$ are real constants.

Taking the inverse Laplace transforms to the equation (2.11), and we obtain the solution for the complex velocity $q$ as,

$$
\begin{aligned}
& q(y, t)=\frac{P_{0}}{\lambda_{1}}\left[\begin{array}{c}
1-e^{-\frac{1}{2} \operatorname{Re} y} \frac{\sinh (a-i b)(1-y)}{\sinh (a-i b)} \\
-e^{\frac{1}{2} \operatorname{Re}(1-y)} \frac{\sinh (a-i b)(y)}{\sinh (a-i b)}
\end{array}\right] \\
& \frac{P_{1}}{\lambda_{1}+i \omega}\left[\begin{array}{l}
1-e^{-\frac{1}{2} \operatorname{Re} y} \frac{\sinh \left(a_{1} \pm i b_{1}\right)(1-y)}{\sinh \left(a_{1} \pm i b_{1}\right)} \\
-e^{\frac{1}{2} \operatorname{Re}(1-y)} \frac{\sinh \left(a_{1} \pm i b_{1}\right)(y)}{\sinh \left(a_{1} \pm i b_{1}\right)}
\end{array}\right] e^{i \omega t} \\
& \frac{\underline{P_{2}}}{\left.\lambda_{1}-\dot{j} \omega\right)}\left[\begin{array}{c}
1-e^{-\frac{1}{2} \operatorname{Re} y} \frac{\sinh \left(a_{2}-i b_{2}\right)(1-y)}{\sinh \left(a_{2}-i b_{2}\right)} \\
-e^{\frac{1}{2} \operatorname{Re}(1-y)} \frac{\sinh \left(a_{2}-i b_{2}\right)(y)}{\sinh \left(a_{2}-i b_{2}\right)}
\end{array}\right] e^{-i \omega t} \\
& -\left(\frac{P_{0}}{\lambda_{1}}+\frac{P_{1}}{\lambda_{1}+i \omega}+\frac{P_{2}}{\lambda_{1}-i \omega}\right) \\
& \text { * }\left[\begin{array}{c}
1-e^{-\frac{1}{2} \operatorname{Re} y} \frac{\sinh (1 / 2) \operatorname{Re}(1-y)}{\sinh (1 / 2) \operatorname{Re}} \\
-e^{\frac{1}{2} \operatorname{Re}(1-y)} \frac{\sinh (1 / 2) \operatorname{Re}(y)}{\sinh (1 / 2) \operatorname{Re}}
\end{array}\right] e^{-\lambda_{1} t} \\
& 2 \sum_{n=1}^{\infty} n \pi\left[(-1)^{n} e^{\frac{1}{2} \operatorname{Re}(1-y)}-e^{-\frac{1}{2} \operatorname{Re}(y)}\right] \\
& +\left(\frac{P_{0}}{s_{1}}+\frac{P_{1}}{s_{1}+i \omega}+\frac{P_{2}}{s_{1}-i \omega}\right) \frac{\sin n \pi y}{\lambda_{1}+s_{1}} e^{s_{1} t}
\end{aligned}
$$

In the equation (2.10), the lower sign is valid for $2 K^{2}>\omega$ and the upper sign is valid for $2 K^{2}<\omega$. The equation (2.13) represents the velocity of the fluid in the general case.

Now we shall consider the following special cases.

Case. 1. Velocity distribution for impulsive pressure gradient:

In this case $P_{1}=P_{2}=0$, then the equation (2.10) reduces to

$$
q(y, t)=\frac{P_{0}}{\lambda_{1}}\left[\begin{array}{c}
1-e^{-\frac{1}{2} \operatorname{Re} y} \frac{\sinh (a-i b)(1-y)}{\sinh (a-i b)} \\
-e^{\frac{1}{2} \operatorname{Re}(1-y)} \frac{\sinh (a-i b)(y)}{\sinh (a-i b)}
\end{array}\right]+
$$


Available online at $w w w . i j r a t . o r g$

$$
\begin{gathered}
-\frac{P_{0}}{\lambda_{1}}\left[\begin{array}{c}
1-e^{-\frac{1}{2} \operatorname{Re} y} \frac{\sinh (1 / 2) \operatorname{Re}(1-y)}{\sinh (1 / 2) \operatorname{Re}} \\
-e^{\frac{1}{2} \operatorname{Re}(1-y)} \frac{\sinh (1 / 2) \operatorname{Re}(y)}{\sinh (1 / 2) \operatorname{Re}}
\end{array}\right] e^{-\lambda_{1} t} \\
+2 \sum_{n=1}^{\infty} n \pi\left[(-1)^{n} e^{\frac{1}{2} \operatorname{Re}(1-y)}-e^{-\frac{1}{2} \operatorname{Re}(y)}\right] \\
\left(\frac{P_{0}}{s_{1}}\right) \frac{\sin n \pi y}{\lambda_{1}+s_{1}} e^{s_{1} t}
\end{gathered}
$$

Case. 2. Velocity distribution for cosine oscillations of pressure gradient:

In this case $P_{0}=0$ and $P_{1}=P_{2}=\frac{P}{2}$, then the equation (2.10) reduces to

$$
\begin{aligned}
& q(y, t)=\frac{P}{2}\left\{\left[\begin{array}{l}
1-e^{-\frac{1}{2} \operatorname{Re} y} \frac{\sinh \left(a_{1} \pm i b_{1}\right)(1-y)}{\sinh \left(a_{1} \pm i b_{1}\right)} \\
-e^{\frac{1}{2} \operatorname{Re}(1-y)} \frac{\sinh \left(a_{1} \pm i b_{1}\right)(y)}{\sinh \left(a_{1} \pm i b_{1}\right)}
\end{array}\right] \frac{e^{i \omega t}}{\lambda_{1}+i \omega}\right. \\
& \left.\left[\begin{array}{l}
1-e^{-\frac{1}{2} \operatorname{Re} y} \frac{\sinh \left(a_{2}-i b_{2}\right)(1-y)}{\sinh \left(a_{2}-i b_{2}\right)} \\
-e^{\frac{1}{2} \operatorname{Re}(1-y)} \frac{\sinh \left(a_{2}-i b_{2}\right)(y)}{\sinh \left(a_{2}-i b_{2}\right)}
\end{array}\right] \frac{e^{-i \omega t}}{\lambda_{1}-i \omega}\right\} \\
& -\frac{P}{2}\left(\frac{1}{\lambda_{1}+i \omega}+\frac{1}{\lambda_{1}-i \omega}\right) \\
& *\left[\begin{array}{c}
1-e^{-\frac{1}{2} \operatorname{Re} y} \frac{\sinh (1 / 2) \operatorname{Re}(1-y)}{\sinh (1 / 2) \operatorname{Re}} \\
-e^{\frac{1}{2} \operatorname{Re}(1-y)} \frac{\sinh (1 / 2) \operatorname{Re}(y)}{\sinh (1 / 2) \operatorname{Re}}
\end{array}\right] e^{-\lambda_{1} t} \\
& +\sum_{n=1}^{\infty} n \pi P\left[(-1)^{n} e^{\frac{1}{2} \operatorname{Re}(1-y)}-e^{-\frac{1}{2} \operatorname{Re}(y)}\right] \\
& \left(\frac{1}{s_{1}+i \omega}+\frac{1}{s_{1}-i \omega}\right) \frac{\sin n \pi y}{\lambda_{1}+s_{1}} e^{s_{1} t}
\end{aligned}
$$

Case. 3. Velocity distribution for sine oscillations of pressure gradient:

$$
\text { In this case } P_{0}=0 \text { and } P_{1}=P_{2}=\frac{P}{2 i} \text {, then }
$$

the equation (2.10) reduces to

$$
\begin{aligned}
& q(y, t)=\frac{P}{2 i}\left\{\left[\begin{array}{c}
1-e^{-\frac{1}{2} \operatorname{Re} y} \frac{\sinh \left(a_{1} \pm i b_{1}\right)(1-y)}{\sinh \left(a_{1} \pm i b_{1}\right)} \\
-e^{\frac{1}{2} \operatorname{Re}(1-y)} \frac{\sinh \left(a_{1} \pm i b_{1}\right)(y)}{\sinh \left(a_{1} \pm i b_{1}\right)}
\end{array}\right] \frac{e^{i \omega t}}{\lambda_{1}+i \omega}\right. \\
& \left.\left[\begin{array}{c}
1-e^{-\frac{1}{2} \operatorname{Re} y} \frac{\sinh \left(a_{2}-i b_{2}\right)(1-y)}{\sinh \left(a_{2}-i b_{2}\right)} \\
-e^{\frac{1}{2} \operatorname{Re}(1-y)} \frac{\sinh \left(a_{2}-i b_{2}\right)(y)}{\sinh \left(a_{2}-i b_{2}\right)}
\end{array}\right] \frac{e^{-i \omega t}}{\lambda_{1}-i \omega}\right\} \\
& -\frac{P}{2 i}\left(\frac{1}{\lambda_{1}+i \omega}+\frac{1}{\lambda_{1}-i \omega}\right) \\
& *\left[\begin{array}{c}
1-e^{-\frac{1}{2} \operatorname{Re} y} \frac{\sinh (1 / 2) \operatorname{Re}(1-y)}{\sinh (1 / 2) \operatorname{Re}} \\
-e^{\frac{1}{2} \operatorname{Re}(1-y)} \frac{\sinh (1 / 2) \operatorname{Re}(y)}{\sinh (1 / 2) \operatorname{Re}}
\end{array}\right] e^{-\lambda_{1} t} \\
& \sum_{n=1}^{\infty} n \pi P i\left[(-1)^{n} e^{\frac{1}{2} \operatorname{Re}(1-y)}-e^{-\frac{1}{2} \operatorname{Re}(y)}\right] \\
& \left(\frac{1}{s_{1}+i \omega}+\frac{1}{s_{1}-i \omega}\right) \frac{\sin n \pi y}{\lambda_{1}+s_{1}} e^{s_{1} t}
\end{aligned}
$$

\section{Shear Stress:}

For the impulsive change of pressure gradient, the nondimensional shear stresses at the wall $y=0$ are given by

$$
\begin{gathered}
\tau_{x}+i \tau_{z}=\left(\frac{\partial q}{\partial y}\right)_{y=0} \\
=\frac{P_{0}}{\lambda_{1}}\left[\begin{array}{l}
\{(1 / 2) \operatorname{Re}+(a-i b)\} \operatorname{coth}(a-i b) \\
+\frac{\{(1 / 2) \operatorname{Re}-(a-i b)\}}{\sinh (a-i b)} e^{\frac{1}{2} \operatorname{Re}}
\end{array}\right] \\
-\frac{P_{0} \operatorname{Re}}{\lambda_{1}} \operatorname{coth} \frac{\operatorname{Re}}{2} e^{-\lambda_{1} t}+ \\
2 \pi^{2} P_{0} \sum_{n=1}^{\infty}\left[(-1)^{n} e^{\frac{1}{2} \operatorname{Re}}-1\right]\left(\frac{n^{2}}{s_{1}}\right) \frac{1}{\lambda_{1}+s_{1}} e^{s_{1} t}
\end{gathered}
$$

For the cosine oscillations of pressure gradient, the nondimensional shear stresses at the wall $y=0$ are given by

$$
\tau_{x}+i \tau_{z}=\left(\frac{\partial q}{\partial y}\right)_{y=0}=
$$




\section{Available online at www.ijrat.org}

$$
\begin{aligned}
& \frac{P}{2}\left\{\begin{array}{c}
\left\{(1 / 2) \operatorname{Re}+\left(a_{1} \pm i b_{1}\right)\right\} \operatorname{coth}\left(a_{1} \pm i b_{1}\right) \\
+\frac{\left\{(1 / 2) \operatorname{Re}-\left(a_{1} \pm i b_{1}\right)\right\}}{\sinh \left(a_{1} \pm i b_{1}\right)} e^{\frac{1}{2} \operatorname{Re}}
\end{array}\right] \frac{e^{i \omega t}}{\lambda_{1}+i \omega} \\
& +\left[\begin{array}{l}
\left.\left\{(1 / 2) \operatorname{Re}+\left(a_{2}-i b_{2}\right)\right\} \operatorname{coth}\left(a_{2}-i b_{2}\right)\right] \\
+\frac{\left\{(1 / 2) \operatorname{Re}-\left(a_{2}-i b_{2}\right)\right\}}{\sinh \left(a_{2}-i b_{2}\right)} e^{\frac{1}{2} \operatorname{Re}}
\end{array}\right] \\
& \left.\frac{e^{-i \omega t}}{\lambda_{1}-i \omega}\right\} \\
& -\frac{P \operatorname{Re}\left(\frac{1}{\lambda_{1}+i \omega}+\frac{1}{\lambda_{1}-i \omega}\right) \operatorname{coth}\left[\frac{1}{2} \operatorname{Re}\right] e^{-\lambda_{1} t}+}{+} \\
& \pi^{2} P \sum_{n=1}^{\infty} n\left[(-1)^{n} e^{\frac{1}{2} \operatorname{Re}}-1\right]\left(\frac{1}{s_{1}+i \omega}+\frac{1}{s_{1}-i \omega}\right) \frac{1}{\lambda_{1}+s_{1}} e^{s_{1} t}
\end{aligned}
$$

For the sine oscillations of pressure gradient, the nondimensional shear stresses at the wall $y=0$ are given by

$$
\begin{aligned}
& \tau_{x}+i \tau_{z}=\left(\frac{\partial q}{\partial y}\right)_{y=0}= \\
& \frac{P}{2 i}\left\{\left[\begin{array}{l}
\left\{(1 / 2) \operatorname{Re}+\left(a_{1} \pm i b_{1}\right)\right\} \operatorname{coth}\left(a_{1} \pm i b_{1}\right) \\
+\frac{\left\{(1 / 2) \operatorname{Re}-\left(a_{1} \pm i b_{1}\right)\right\}}{\sinh \left(a_{1} \pm i b_{1}\right)} e^{\frac{1}{2} \operatorname{Re}}
\end{array}\right] \frac{e^{i \omega t}}{\lambda_{1}+i \omega}\right. \\
& +\left[\begin{array}{l}
\left\{(1 / 2) \operatorname{Re}+\left(a_{2}-i b_{2}\right)\right\} \operatorname{coth}\left(a_{2}-i b_{2}\right) \\
+\frac{\left\{(1 / 2) \operatorname{Re}-\left(a_{2}-i b_{2}\right)\right\}}{\sinh \left(a_{2}-i b_{2}\right)} e^{\frac{1}{2} \operatorname{Re}}
\end{array}\right] \\
& \left.\frac{e^{-i \omega t}}{\lambda_{1}-i \omega}\right\} \\
& -\frac{P \operatorname{Re}}{2 i}\left(\frac{1}{\lambda_{1}+i \omega}+\frac{1}{\lambda_{1}-i \omega}\right) \operatorname{coth}\left[\frac{1}{2} \operatorname{Re}\right] e^{-\lambda_{1} t} \\
& \pi^{2} P \sum_{n=1}^{\infty} n\left[(-1)^{n} e^{\frac{1}{2} \operatorname{Re}}-1\right] \\
& +\left(\frac{1}{s_{1}+i \omega}+\frac{1}{s_{1}-i \omega}\right) \frac{1}{\lambda_{1}+s_{1}} e^{s_{1} t}
\end{aligned}
$$

\section{RESULTS AND DISCUSSION}

The unsteady MHD flow of a viscous incompressible electrically conducting fluid through porous medium in a rotating parallel plate channel with variable pressure gradient has been studied. The governing equations are solved analytically using the Laplace transform technique. For computationally we have considered three different cases 1 . Impulsive change of pressure gradient, 2. Cosine oscillations of pressure gradient and 3. Sine oscillations of pressure gradient. The flow governed by the non-dimensional parameters for the velocity components $u$ and $w$ with different values of magnetic parameter $M$, rotation parameter $K$, Reynolds number Re, $D$ the permeability parameter, frequency parameter $\omega$ and phage angle $\omega t$ Fig.2-Fig.19 represents the velocity profiles for impulsive pressure gradient Fig.10-Fig.21 represent the velocity profiles for cosine oscillations of pressure gradient, where as the Fig.22-Fig.33 represent the velocity profiles for sine oscillations of pressure gradient.

We have seen from Fig.2, Fig.10 and Fig.22 that the velocity component $u$ increases with an increase in magnetic parameter $M$ for the impulsive change, and decreases for cosine and sine oscillations of the pressure gradient. Fig.3, Fig.11 and Fig.23 shows that the velocity component $w$ decreases for the cosine oscillations of the pressure gradient while it increases for impulsive change and sine oscillations of the pressure gradient with an increase in magnetic parameter $M$. As expected due to the fact that the application of transverse magnetic field results to a resistive type force (called Lorentz force) similar to drag force and upon increasing the values of magnetic parameter, the drag force increases which leads to the deceleration of the flow. It is seen from Fig.4, Fig.5, Fig. 12 and Fig.13 that the primary velocity $u$ and the secondary velocity $w$ decreases for cosine oscillations of the pressure gradient, while primary velocity $u$ increases and $w$ reduces throughout the fluid region with an increase in rotation parameter $K$ for impulse change, the reversal behaviour is observed for sine oscillations of the pressure gradient. The rotation parameter defines the relative magnitude of the Coriolis force and the viscous force in the regime; therefore it is clear that high magnitude Coriolis forces are counterproductive for the primary flow. Fig.6, Fig.14 and Fig.26, we noticed that the primary velocity $u$ decreases with an increase in Reynolds number $\mathrm{Re}$ for the impulsive change, while it increases with an increase in Reynolds number $\operatorname{Re}$ for cosine oscillations of the pressure gradient, the magnitude of the velocity component $w$ enhances initially for $y \leq 0.2$ and then gradually reduces for $0.4 \leq y \leq 1$ with an increase in Reynolds number $\mathrm{Re}$ for sine oscillations of the pressure gradient.

It is seen from Fig.7, Fig.15 and Fig.27 that the secondary velocity $w$ increases for the impulsive change while it decreases for sine oscillations of the pressure gradient with an increase in Reynolds number Re. Similarly, the magnitude of the velocity component $w$ enhances initially for $y \leq 0.2$ and then gradually reduces for $0.2<y \leq 1$ with an increase in Reynolds 


\section{Available online at www.ijrat.org}

number Re for cosine oscillations of the pressure gradient. It is seen from Fig.8, Fig.16, and Fig.28 that the primary velocity $u$ increases with an increase in permeability parameter $D$ for the impulsive change, cosine and sine oscillations of the pressure gradient throughout the fluid region. Likewise from Fig.9, Fig.17 and Fig.29 that the secondary velocity $w$ reduces with an increase in permeability parameter $D$ for sine oscillations of the pressure gradient while it enhances for the impulsive change and cosine oscillations of the pressure gradient. Fig.18 and Fig.30 shown that the primary velocity $u$ increases with an increase in frequency parameter $\omega$ for cosine oscillations of the pressure gradient while the velocity $u$ enhances for $\omega=2,4 \& 6$ and then experiences retardation for $\omega=8$ for sine oscillations of the pressure gradient. It has been seen from Fig.15 and Fig.31 that the secondary velocity $w$ decreases for the sine oscillations of the pressure gradient while the velocity $u$ enhances for $\omega=2,4 \& 6$ and then experiences retardation for $\omega=8$ for cosine oscillations of the pressure gradient with an increase in frequency parameter $\omega$. Finally we have noticed from Fig.20 and Fig.32 that, the magnitude of the primary velocity $u$ decreases with an increase in phase angle $\omega t$ for both cosine and sine oscillations of the pressure gradient. It is also seen from Fig. 21 and Fig. 33 that the secondary velocity $w$ reduces for cosine oscillations of the pressure gradient while it increases for sine oscillations of the pressure gradient with an increase in phase angle $\omega t$. It is noted that, the magnitude of the velocities for cosine oscillations of the pressure gradient are always greater than magnitude of sine oscillations of the pressure gradient.

The non-dimensional shear stresses $\tau_{x}$ and $\tau_{z}$ have been calculated at the wall $(y=0)$ due to the primary and the secondary flows are presented in Table1-Table6 and computationally discussed with reference to governing parameters like, magnetic parameter $M$, rotation parameter $K$, Reynolds number Re, Permeability parameter $D$, frequency parameter $\omega$ and phase angle $\omega t$. We notice that the shear stresses

\section{GRAPHS \& TABLES}

I. Velocity Profiles with Impulsive Pressure Gradient:

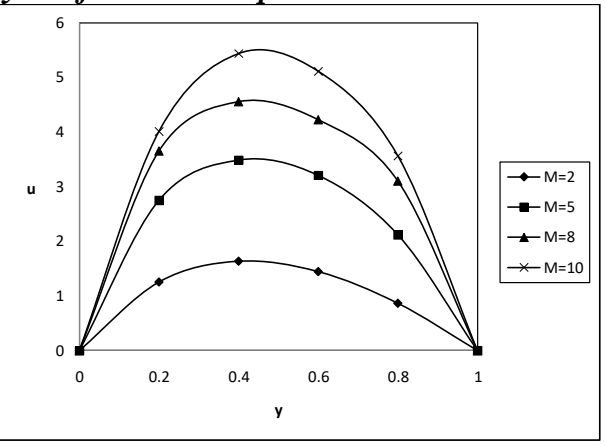

Fig. 2: The velocity Profile for $u$ against $M$ with $D=1, K=2, \operatorname{Re}=2, m=1, t=0.1, P_{0}=10$ $\tau_{x}$ and $\tau_{z}$ due to the primary and secondary flow at the wall $y=0$ reduce for the impulsive change, cosine and sine oscillations of the pressure gradient with an increase in Hartmann number $M$. The magnitude of the shear stress $\tau_{x}$ due to the primary flow decreases for the impulsive change and cosine oscillations, while it increases for sine oscillations of the pressure gradient with an increase in rotation parameter $K$ or Permeability parameter $D$. It is found that the shear stress $\tau_{z}$ decreases for both impulsive change and cosine oscillations of the pressure gradient while it increases for sine oscillations of the pressure gradient with an increase in rotation parameter $K$ and Permeability parameter $D$. The shear stress $\tau_{x}$ decreases for small values of magnetic parameter $M(\leq 8)$ and then it increases for the impulsive change, cosine and sine oscillations of the pressure gradient with an increase in Reynolds number Re. Also the shear stress $\tau_{z}$ increases for both impulsive change and cosine oscillations of the pressure gradient while it decreases for sine oscillations of the pressure gradient with an increase in Reynolds number Re. The shear stress $\tau_{x}$ increases for small values of magnetic parameter $M(\leq 8)$ and then it decreases for cosine and sine oscillations of the pressure gradient with an increase in frequency parameter $\omega$. It is found that the shear stress $\tau_{z}$ increases for small values of magnetic parameter $M(\leq 8)$ and then it decreases for cosine oscillations while it first decreases and then increases for sine oscillations of the pressure gradient with an increase in frequency parameter $\omega$.

The shear stress $\tau_{x}$ decreases for both cosine and sine oscillations of the pressure gradient with an increase in phage angle $\omega t$. Also the shear stress $\tau_{z}$ decreases for cosine oscillations of the pressure gradient while it increases for sine oscillations of the pressure gradient with an increase in phage angle $\omega t$.

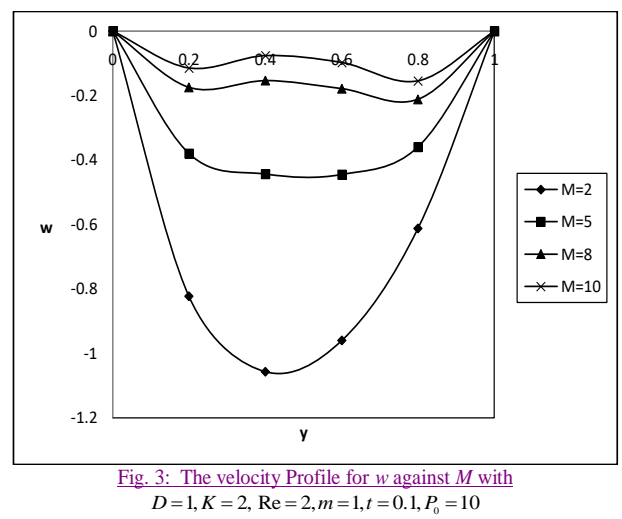


International Journal of Research in Advent Technology, Vol.7, No.4, April 2019 E-ISSN: 2321-9637

Available online at www.ijrat.org
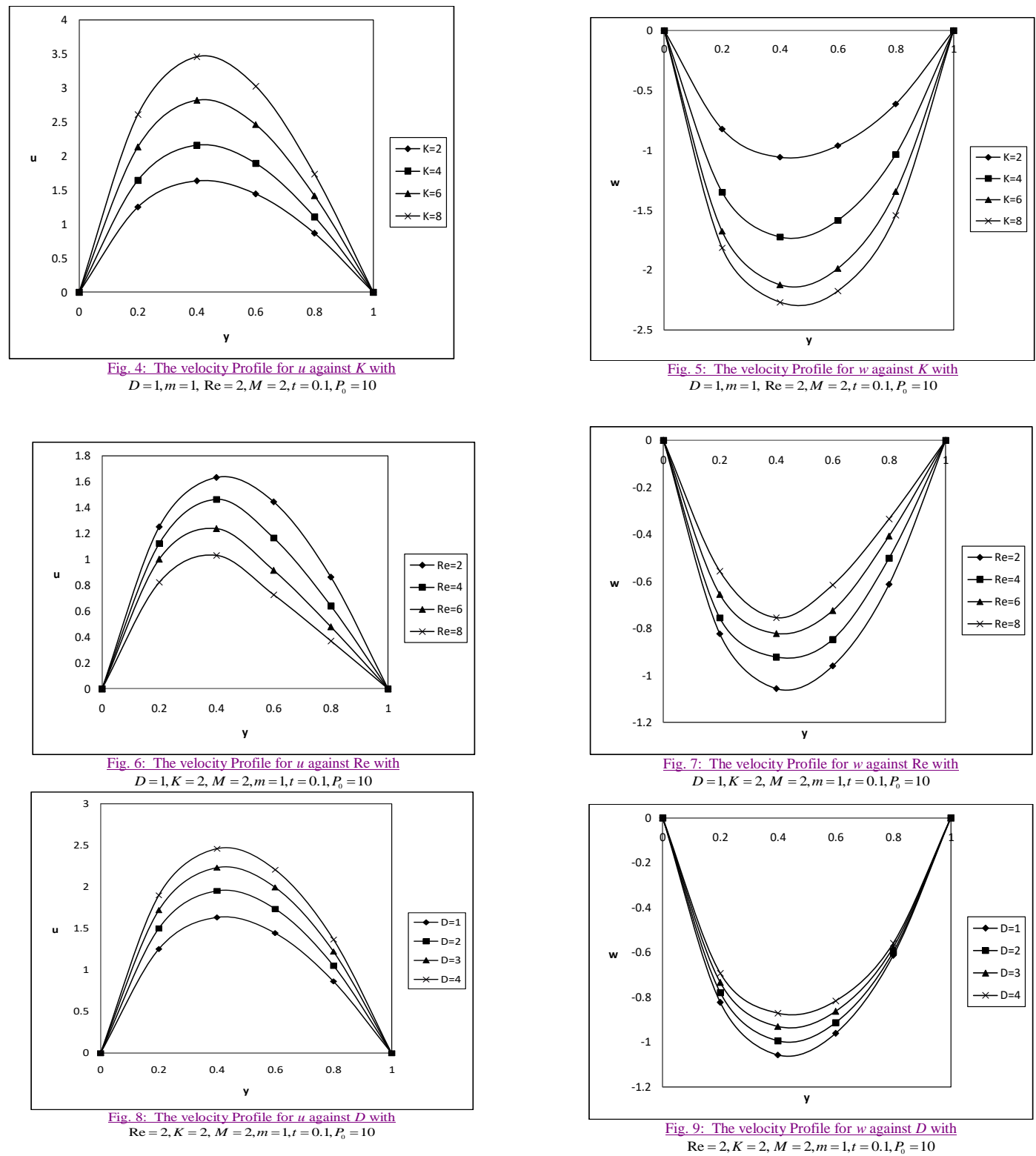

II. Velocity Profiles with Cosine Oscillation of Pressure Gradient:

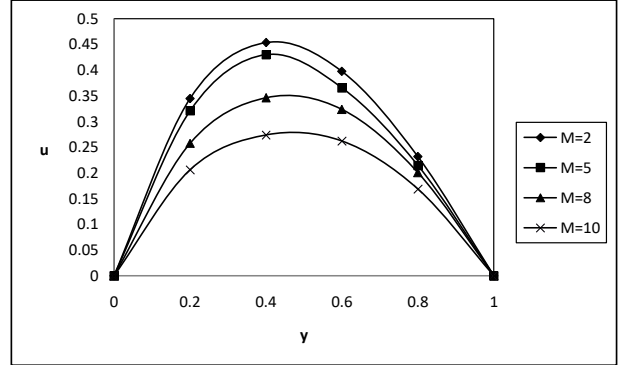

Fig. 10: The velocity Profile for $u$ against $M$ with

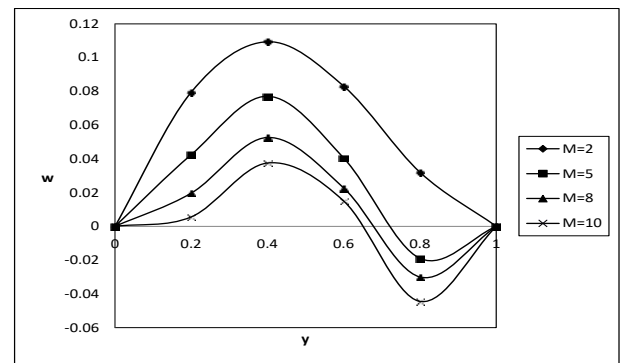

Fig. 11: The velocity Profile for $w$ against $M$ with
$D=1, K=2, \operatorname{Re}=2, m=1, \omega=2, \omega t=\pi / 4, t=0.1, P_{0}=10$ 
International Journal of Research in Advent Technology, Vol.7, No.4, April 2019 E-ISSN: 2321-9637

Available online at www.ijrat.org

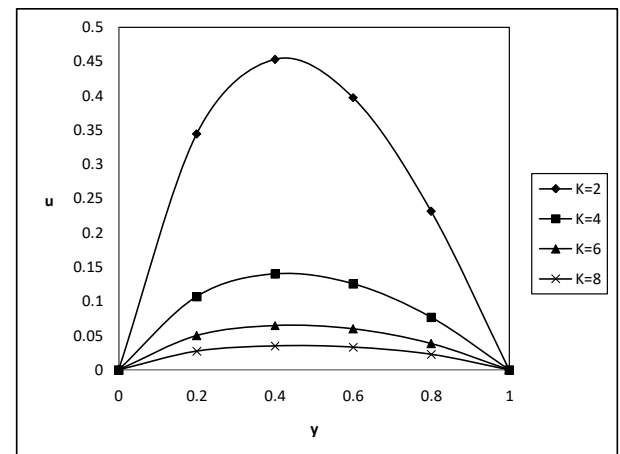

Fig. 12: The velocity Profile for $u$ against $K$ with

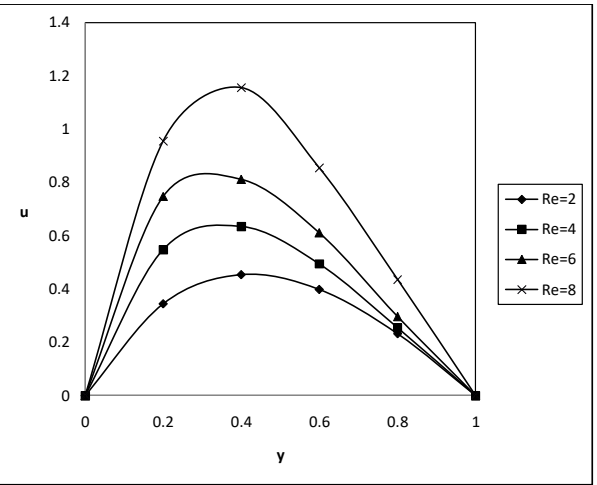

Fig. 14: The velocity Profile for $u$ against Re with

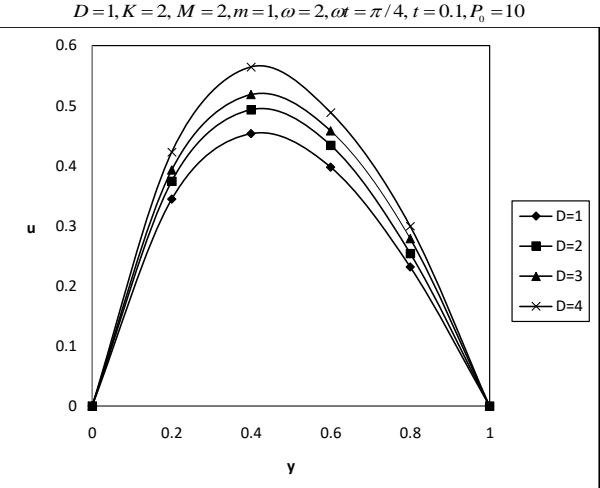

Fig. 16: The velocity Profile for $u$ against $D$ with

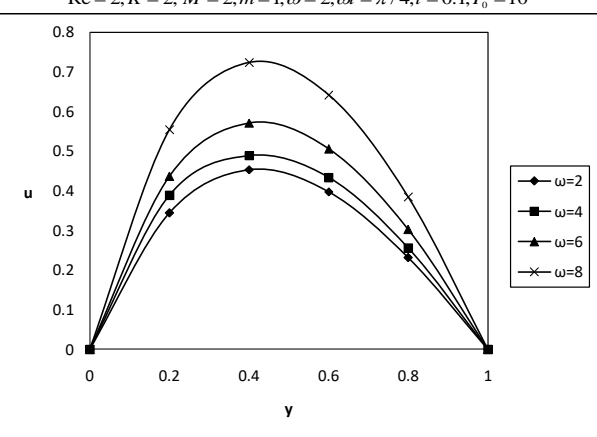

Fig. 18: The velocity Profile for $u$ against $D$ with

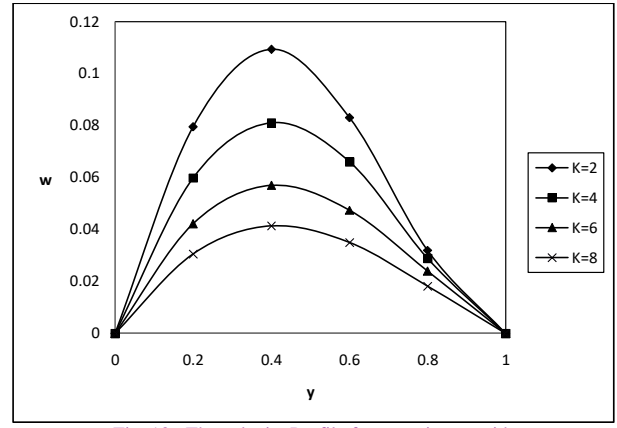

Fig. 13: The velocity Profile for $w$ against $K$ with

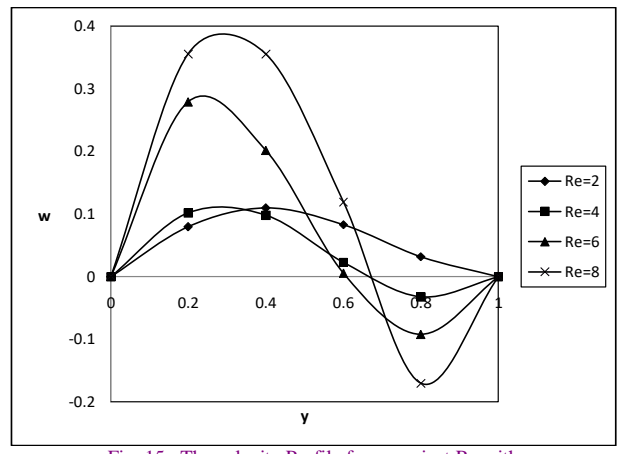

Fig. 15: The velocity Profile for $w$ against Re with
$D=1, K=2, M=2, m=1, \omega=2, \omega t=\pi / 4, t=0.1, P_{0}=10$

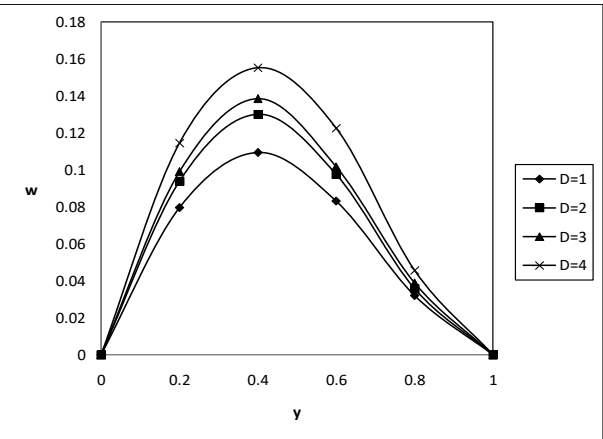

Fig. 17: The velocity Profile for $w$ against $D$ with

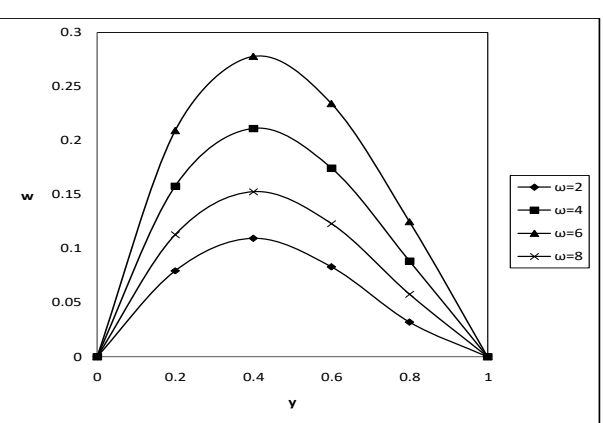

Fig. 19: The velocity Profile for $w$ against $D$ with
$\operatorname{Re}=2, K=2, M=2, m=1, \omega t=\pi / 4, t=0.1, P_{0}=10$ 
International Journal of Research in Advent Technology, Vol.7, No.4, April 2019 E-ISSN: 2321-9637

Available online at www.ijrat.org

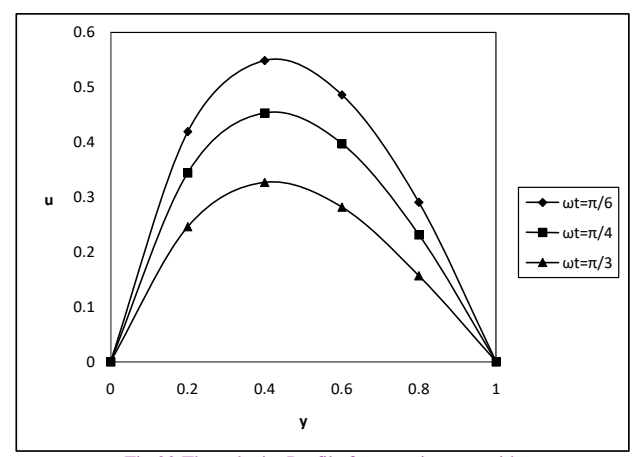

Fig.20 The velocity Profile for $u$ against $\omega t$ with
$\operatorname{Re}=2, K=2, M=2, m=1, \omega=2, t=0.1, P_{0}=10$

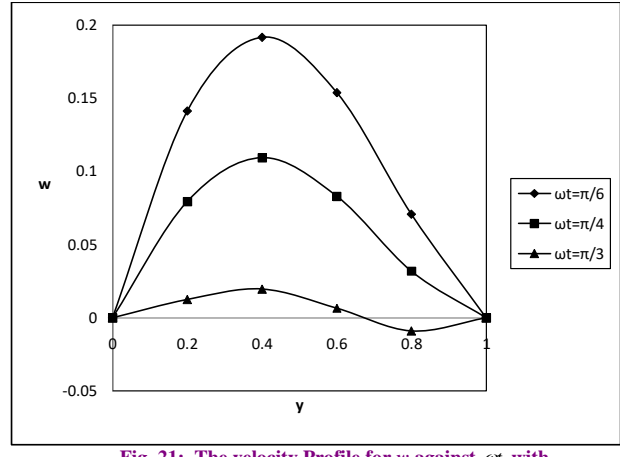

Fig. 21: The velocity Profile for $w$ against $\frac{\omega t}{w}$ with $\operatorname{Re}=2, K=2, M=2, m=1, \omega=2, t=0.1, P_{0}=10$

III. Velocity Profiles with Sine Oscillation of Pressure Gradient:

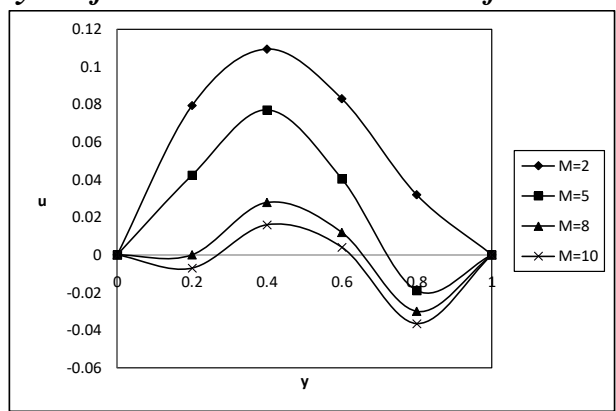

Fig. 22: The velocity Profile for $u$ against $M$ with

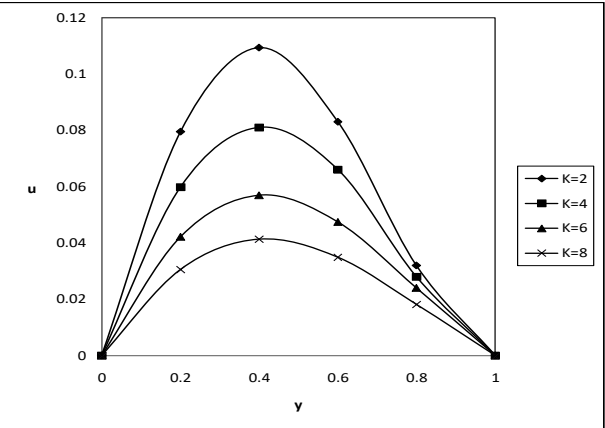

Fig. 24: The velocity Profile for $u$ against $K$ with
$D=1, m=1, \operatorname{Re}=2, M=2, \omega=2, \omega t=\pi / 4, t=0.1, P_{0}=10$

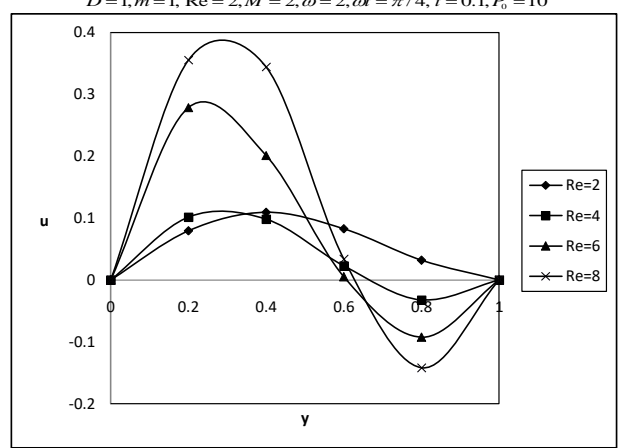

Fig. 26: The velocity Profile for $u$ against Re with
$D=1, K=2, M=2, m=1, \omega=2, \omega t=\pi / 4, t=0.1, P_{0}=10$

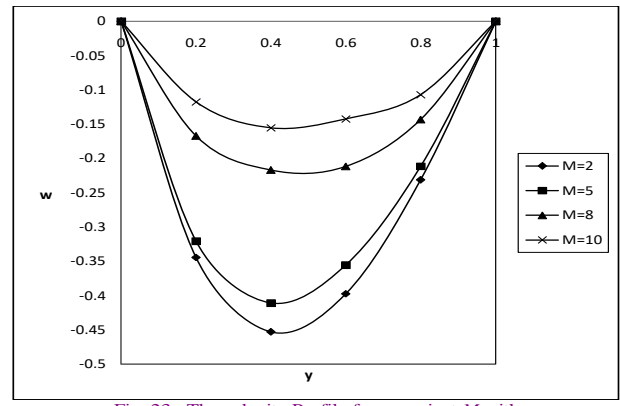

Fig. 23: The velocity Profile for $w$ against $M$ with
$D=1, K=2, \operatorname{Re}=2, m=1, \omega=2, \omega t=\pi / 4, t=0.1, P_{0}=10$

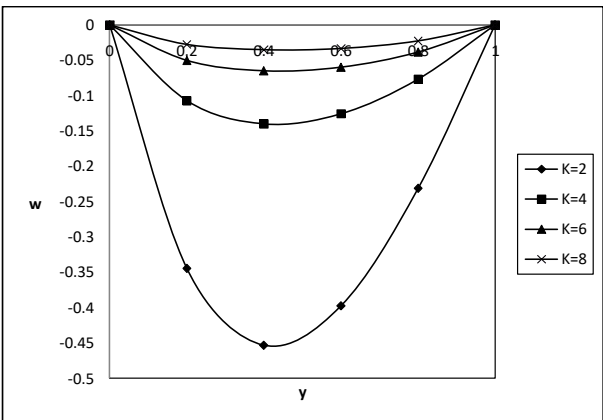

Fig. 25: The velocity Profile for $w$ against $K$ with

$D=1, m=1, \operatorname{Re}=2, M=2, \omega=2, \omega t=\pi / 4, t=0.1, P_{0}=10$

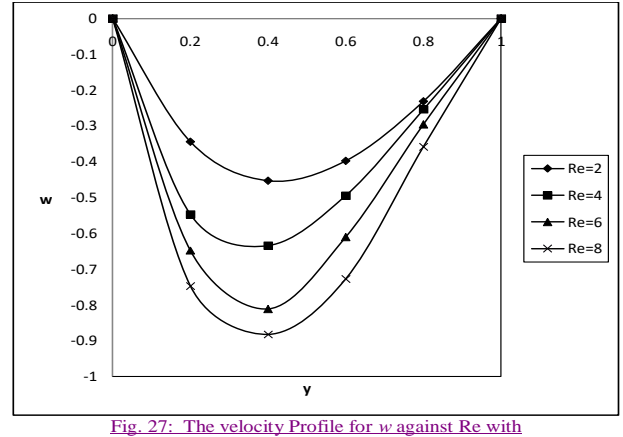

Fig. 27: The velocity Profile for $w$ against Re with
$D=1, K=2, M=2, m=1, \omega=2, \omega t=\pi / 4, t=0.1, P_{0}=10$ 
International Journal of Research in Advent Technology, Vol.7, No.4, April 2019 E-ISSN: 2321-9637

Available online at www.ijrat.org

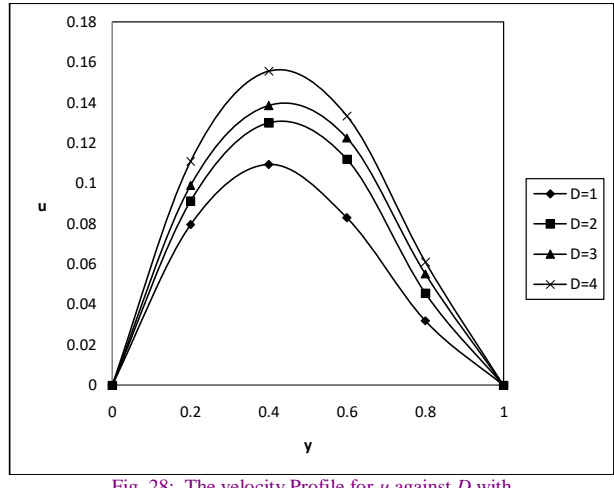

Fig. 28: The velocity Profile for $u$ against $D$ with

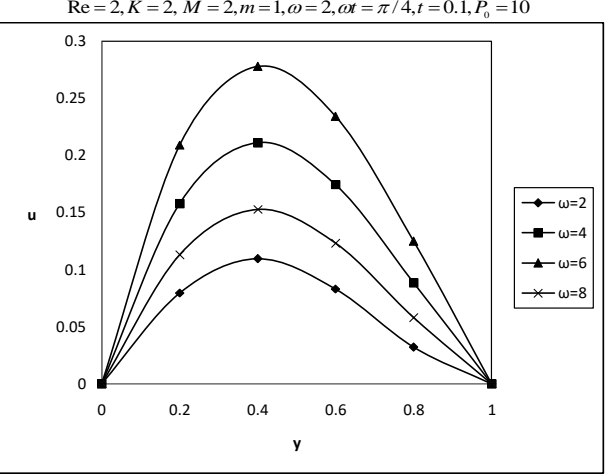

Fig. 30: The velocity Profile for $u$ against $D$ with
$\operatorname{Re}=2, K=2, D=1, M=2, m=1, \omega t=\pi / 4, t=0.1, P_{0}$

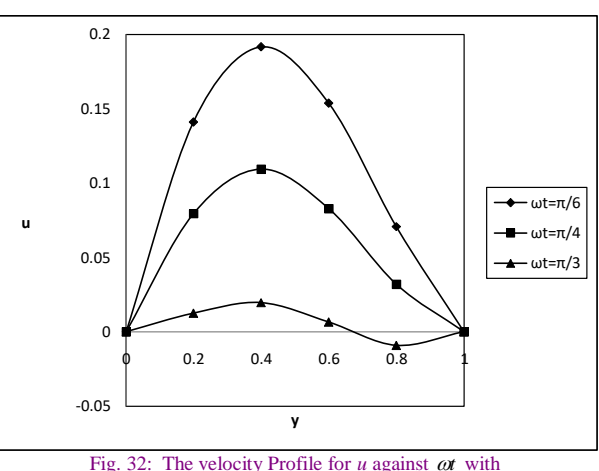

$\operatorname{Re}=2, K=2, M=2, m=1, \omega=2, t=0.1, P_{0}=10$

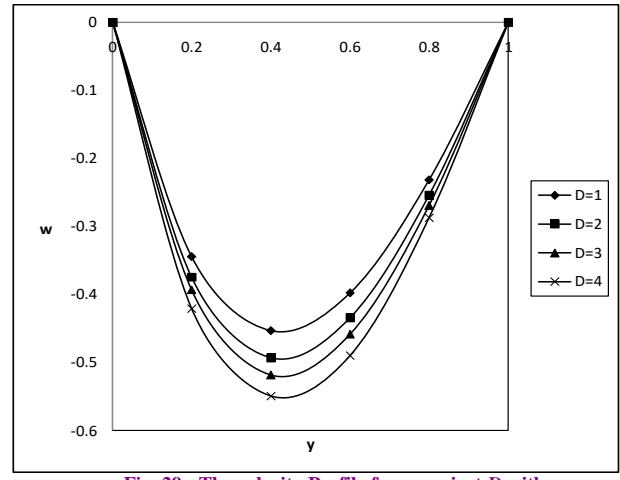

Fig. 29: The velocity Profile for $w$ against $D$ with
$\operatorname{Re}=2, K=2, M=2, m=1, \omega=2, \omega t=\pi / 4, t=0.1, P_{0}=10$

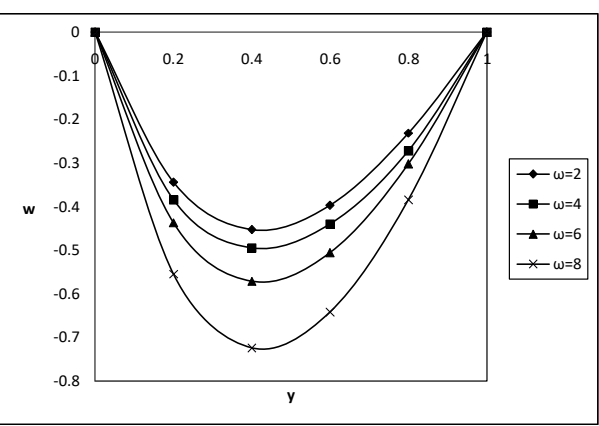

Fig. 31: The velocity Profile for $w$ against $D$ with
$\operatorname{Re}=2, K=2, M=2, m=1, \omega t=\pi / 4, t=0.1, P_{0}=10$

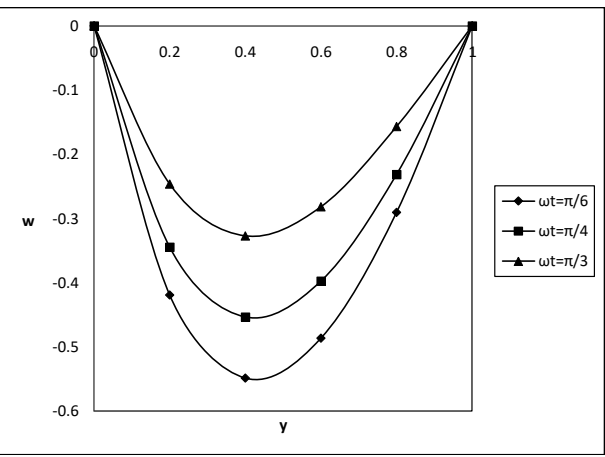

Fig. 33: The velocity Profile for $w$ against $\omega t$ with $\operatorname{Re}=2, K=2, M=2, m=1, \omega=2, t=0.1, P_{0}=10$

\begin{tabular}{|c|c|c|c|c|c|c|c|}
\hline & $K$ & $\mathrm{R} e$ & $D$ & $\mathrm{M}=2$ & $\mathrm{M}=5$ & $\mathrm{M}=8$ & $\mathrm{M}=10$ \\
\hline I & 2 & 2 & 1 & 0.920545 & 0.755471 & 0.544854 & 0.388507 \\
\hline II & 4 & 2 & 1 & 0.901254 & 0.722564 & 0.522541 & 0.355689 \\
\hline III & 6 & 2 & 1 & 0.887459 & 0.700142 & 0.500245 & 0.445088 \\
\hline IV & 2 & 4 & 1 & 0.785540 & 0.588914 & 0.366982 & 0.455212 \\
\hline V & 2 & 6 & 1 & 0.678801 & 0.321415 & 0.288795 & 0.482546 \\
\hline VI & 2 & 2 & 2 & 0.855852 & 0.624558 & 0.323266 & 0.211452 \\
\hline VII & 2 & 2 & 3 & 0.648878 & 0.405525 & 0.200145 & 0.144523 \\
\hline
\end{tabular}

Table 1: The shear stress $\left(\tau_{x}\right)$ at the wall $y=0$ with impulsive pressure gradient

\begin{tabular}{|c|c|c|c|c|c|c|c|}
\hline & $K$ & $\mathrm{R} e$ & $D$ & $\mathrm{M}=2$ & $\mathrm{M}=5$ & $\mathrm{M}=8$ & $\mathrm{M}=10$ \\
\hline I & 2 & 2 & 1 & 0.566585 & 0.510225 & 0.478845 & 0.425563 \\
\hline II & 4 & 2 & 1 & 0.522142 & 0.488574 & 0.422548 & 0.388546 \\
\hline III & 6 & 2 & 1 & 0.500214 & 0.466506 & 0.399564 & 0.332564 \\
\hline IV & 2 & 4 & 1 & 0.788548 & 0.622564 & 0.578045 & 0.488574 \\
\hline V & 2 & 6 & 1 & 0.966569 & 0.745805 & 0.655482 & 0.540478 \\
\hline VI & 2 & 2 & 2 & 0.704458 & 0.615542 & 0.588478 & 0.522453 \\
\hline VII & 2 & 2 & 3 & 0.800145 & 0.711214 & 0.652546 & 0.599698 \\
\hline
\end{tabular}

Table 2: The shear stress $\left(\tau_{z}\right)$ at the wall $y=0$ with impulsive pressure gradient 
International Journal of Research in Advent Technology, Vol.7, No.4, April 2019 E-ISSN: 2321-9637

Available online at www.ijrat.org

\begin{tabular}{|c|c|c|c|c|c|c|c|c|c|}
\hline & $K$ & $\mathrm{R} e$ & $D$ & $\omega$ & $\omega t$ & $\mathrm{M}=2$ & $\mathrm{M}=5$ & $\mathrm{M}=8$ & $\mathrm{M}=10$ \\
\hline $\mathrm{I}$ & 2 & 2 & 1 & 2 & $\pi / 4$ & 0.814025 & 0.755845 & 0.681452 & 0.622142 \\
\hline $\mathrm{II}$ & 4 & 2 & 1 & 2 & $\pi / 4$ & 0.722459 & 0.699857 & 0.640563 & 0.574486 \\
\hline $\mathrm{III}$ & 6 & 2 & 1 & 2 & $\pi / 4$ & 0.622546 & 0.584740 & 0.589902 & 0.510252 \\
\hline $\mathrm{IV}$ & 2 & 4 & 1 & 2 & $\pi / 4$ & 0.788458 & 0.685548 & 0.622548 & 0.684476 \\
\hline $\mathrm{V}$ & 2 & 6 & 1 & 2 & $\pi / 4$ & 0.688982 & 0.566809 & 0.566527 & 0.722105 \\
\hline $\mathrm{VI}$ & 2 & 2 & 2 & 2 & $\pi / 4$ & 0.699885 & 0.685041 & 0.661245 & 0.600732 \\
\hline $\mathrm{VII}$ & 2 & 2 & 3 & 2 & $\pi / 4$ & 0.588987 & 0.562215 & 0.541046 & 0.522146 \\
\hline $\mathrm{VIII}$ & 2 & 2 & 1 & 4 & $\pi / 4$ & 0.998702 & 0.855406 & 0.746025 & 0.520214 \\
\hline $\mathrm{IX}$ & 2 & 2 & 1 & 6 & $\pi / 4$ & 1.225473 & 0.958456 & 0.866502 & 0.445106 \\
\hline $\mathrm{X}$ & 2 & 2 & 1 & 2 & $\pi / 6$ & 0.885442 & 0.822546 & 0.755480 & 0.688549 \\
\hline $\mathrm{XI}$ & 2 & 2 & 1 & 2 & $\pi / 3$ & 0.745582 & 0.722152 & 0.622548 & 0.588479 \\
\hline
\end{tabular}

Table 3: The shear stress $\left(\tau_{x}\right)$ at the wall $y=0$ with cosine oscillations of pressure gradient

\begin{tabular}{|c|c|c|c|c|c|c|c|c|c|}
\hline & $K$ & $\mathrm{R} e$ & $D$ & $\omega$ & $\omega t$ & $\mathrm{M}=2$ & $\mathrm{M}=5$ & $\mathrm{M}=8$ & $\mathrm{M}=10$ \\
\hline $\mathrm{I}$ & 2 & 2 & 1 & 2 & $\pi / 4$ & 0.662152 & 0.622154 & 0.596655 & 0.577458 \\
\hline $\mathrm{II}$ & 4 & 2 & 1 & 2 & $\pi / 4$ & 0.604252 & 0.566254 & 0.533624 & 0.511425 \\
\hline $\mathrm{III}$ & 6 & 2 & 1 & 2 & $\pi / 4$ & 0.541125 & 0.522143 & 0.499854 & 0.466233 \\
\hline $\mathrm{IV}$ & 2 & 4 & 1 & 2 & $\pi / 4$ & 0.725546 & 0.688549 & 0.622504 & 0.599685 \\
\hline $\mathrm{V}$ & 2 & 6 & 1 & 2 & $\pi / 4$ & 0.788544 & 0.722145 & 0.685502 & 0.622115 \\
\hline $\mathrm{VI}$ & 2 & 2 & 2 & 2 & $\pi / 4$ & 0.755066 & 0.699584 & 0.658956 & 0.602156 \\
\hline $\mathrm{VII}$ & 2 & 2 & 3 & 2 & $\pi / 4$ & 0.822103 & 0.755263 & 0.702023 & 0.655246 \\
\hline $\mathrm{VIII}$ & 2 & 2 & 1 & 4 & $\pi / 4$ & 0.702254 & 0.665445 & 0.622542 & 0.500246 \\
\hline $\mathrm{IX}$ & 2 & 2 & 1 & 6 & $\pi / 4$ & 0.722546 & 0.705871 & 0.655589 & 0.455213 \\
\hline $\mathrm{X}$ & 2 & 2 & 1 & 2 & $\pi / 6$ & 0.622352 & 0.602056 & 0.574856 & 0.550026 \\
\hline $\mathrm{XI}$ & 2 & 2 & 1 & 2 & $\pi / 3$ & 0.600214 & 0.586966 & 0.552466 & 0.522336 \\
\hline
\end{tabular}

Table 4: The shear stress $\left(\tau_{z}\right)$ at the wall $y=0$ with cosine oscillations of pressure gradient

\begin{tabular}{|c|c|c|c|c|c|c|c|c|c|}
\hline & $K$ & $\mathrm{R} e$ & $D$ & $\omega$ & $\omega t$ & $\mathrm{M}=2$ & $\mathrm{M}=5$ & $\mathrm{M}=8$ & $\mathrm{M}=10$ \\
\hline $\mathrm{I}$ & 2 & 2 & 1 & 2 & $\pi / 4$ & 0.356636 & 0.322546 & 0.299555 & 0.277485 \\
\hline $\mathrm{II}$ & 4 & 2 & 1 & 2 & $\pi / 4$ & 0.388546 & 0.352262 & 0.314452 & 0.299873 \\
\hline $\mathrm{III}$ & 6 & 2 & 1 & 2 & $\pi / 4$ & 0.399658 & 0.366585 & 0.322506 & 0.302145 \\
\hline $\mathrm{IV}$ & 2 & 4 & 1 & 2 & $\pi / 4$ & 0.321021 & 0.302589 & 0.288695 & 0.266899 \\
\hline $\mathrm{V}$ & 2 & 6 & 1 & 2 & $\pi / 4$ & 0.301332 & 0.284585 & 0.266554 & 0.244855 \\
\hline $\mathrm{VI}$ & 2 & 2 & 2 & 2 & $\pi / 4$ & 0.366525 & 0.336502 & 0.300045 & 0.299845 \\
\hline $\mathrm{VII}$ & 2 & 2 & 3 & 2 & $\pi / 4$ & 0.377785 & 0.344215 & 0.321787 & 0.332566 \\
\hline $\mathrm{VIII}$ & 2 & 2 & 1 & 4 & $\pi / 4$ & 0.255466 & 0.210023 & 0.199665 & 0.156656 \\
\hline $\mathrm{IX}$ & 2 & 2 & 1 & 6 & $\pi / 4$ & 0.221455 & 0.188545 & 0.144526 & 0.122546 \\
\hline $\mathrm{X}$ & 2 & 2 & 1 & 2 & $\pi / 6$ & 0.422515 & 0.400526 & 0.355626 & 0.322145 \\
\hline $\mathrm{XI}$ & 2 & 2 & 1 & 2 & $\pi / 3$ & 0.333256 & 0.302214 & 0.255664 & 0.221552 \\
\hline
\end{tabular}

Table 5: The shear stress $\left(\tau_{x}\right)$ at the wall $y=0$ with sine oscillations of pressure gradient 
International Journal of Research in Advent Technology, Vol.7, No.4, April 2019

$$
\text { E-ISSN: 2321-9637 }
$$

Available online at www.ijrat.org

\begin{tabular}{|c|c|c|c|c|c|c|c|c|c|}
\hline & $K$ & $\mathrm{R} e$ & $D$ & $\omega$ & $\omega t$ & $\mathrm{M}=2$ & $\mathrm{M}=5$ & $\mathrm{M}=8$ & $\mathrm{M}=10$ \\
\hline $\mathrm{I}$ & 2 & 2 & 1 & 2 & $\pi / 4$ & 0.278843 & 0.255655 & 0.233652 & 0.201145 \\
\hline $\mathrm{II}$ & 4 & 2 & 1 & 2 & $\pi / 4$ & 0.296585 & 0.272562 & 0.255626 & 0.228545 \\
\hline $\mathrm{III}$ & 6 & 2 & 1 & 2 & $\pi / 4$ & 0.322145 & 0.298845 & 0.275699 & 0.250142 \\
\hline $\mathrm{IV}$ & 2 & 4 & 1 & 2 & $\pi / 4$ & 0.222541 & 0.200145 & 0.188547 & 0.166585 \\
\hline $\mathrm{V}$ & 2 & 6 & 1 & 2 & $\pi / 4$ & 0.201145 & 0.184078 & 0.144585 & 0.122548 \\
\hline $\mathrm{VI}$ & 2 & 2 & 2 & 2 & $\pi / 4$ & 0.325266 & 0.301225 & 0.285596 & 0.255896 \\
\hline $\mathrm{VII}$ & 2 & 2 & 3 & 2 & $\pi / 4$ & 0.350021 & 0.322546 & 0.302152 & 0.288549 \\
\hline $\mathrm{VIII}$ & 2 & 2 & 1 & 4 & $\pi / 4$ & 0.254025 & 0.233256 & 0.201232 & 0.178858 \\
\hline $\mathrm{IX}$ & 2 & 2 & 1 & 6 & $\pi / 4$ & 0.313365 & 0.299585 & 0.255485 & 0.222215 \\
\hline $\mathrm{X}$ & 2 & 2 & 1 & 2 & $\pi / 6$ & 0.352662 & 0.252266 & 0.200214 & 0.155242 \\
\hline $\mathrm{XI}$ & 2 & 2 & 1 & 2 & $\pi / 3$ & 0.399685 & 0.370254 & 0.325650 & 0.256654 \\
\hline
\end{tabular}

Table 6: The shear stress $\left(\tau_{z}\right)$ at the wall $y=0$ with sine oscillations of pressure gradient

\section{CONCLUSIONS:}

* The velocity component for primary flow enhances with increasing $M, K$ and $D$, and reduces with $\mathrm{Re}$ for the impulsive change of pressure gradient.

* The velocity component for secondary flow enhances with increasing $M, \operatorname{Re}$ and $D$, and reduces with $\mathrm{K}$ for the impulsive change of pressure gradient.

* The velocity component for primary flow increases with increasing $\operatorname{Re}, D$ and $\omega$, and reduces with $M, K$ and phase angle $\omega t$ for the cosine oscillations of pressure gradient.

* The velocity component for primary flow increases with increasing $D$, and reduces with $M, K$ and phase angle $\omega t$ for the sine oscillations of pressure gradient.

* The magnitude of the velocity components for primary flow and for secondary flow enhances initially for $y \leq 0.2$ and then gradually reduces for $0.2<y \leq 1$ with an increase in Reynolds number $\mathrm{Re}$ for sine and cosine oscillations of the pressure gradient respectively.

* The velocity for primary flow and for secondary flow enhances for $\omega=2,4 \& 6$ and then experiences retardation for $\omega=8$ for sine and cosine oscillations of the pressure gradient respectively.

* The velocity component for secondary flow enhances with increasing $M, K$ and phase angle $\omega t$, and reduces with increase in $\operatorname{Re}, D$ and frequency of oscillation $\omega$ for the impulsive change of pressure gradient.

* The magnitude of the shear stress $\tau_{x}$ due to the primary flow decreases for the impulsive change and cosine of the pressure gradient with an increase in $M, \operatorname{Re}, K$ or Permeability parameter $D$.

* The magnitude of the shear stress $\tau_{z}$ due to the secondary flow reduces for the impulsive change and cosine of the pressure gradient with an increase in rotation parameter $M$ or $K$ and enhances with increasing in $\operatorname{Re}$ or $D$.

* Both the stresses enhance with increase in $K$ and $D$; and reduce with increase in $M$ or Re for sine of the pressure gradient.

* The shear stress $\tau_{x}$ increases for small values of magnetic parameter $M(\leq 8)$ and then it decreases for cosine and sine oscillations of the pressure gradient with an increase in frequency parameter $\omega$.

* The shear stress $\tau_{z}$ increases for small values of magnetic parameter $M(\leq 8)$ and then it decreases for cosine oscillations while it first decreases and then increases for sine oscillations of the pressure gradient with an increase in frequency parameter $\omega$.

* The shear stress $\tau_{x}$ decreases for both cosine and sine oscillations of the pressure gradient with an increase in phage angle $\omega t$. Also the shear stress $\tau_{z}$ decreases for cosine oscillations of the pressure gradient while it increases for sine oscillations of the pressure gradient with an increase in phage angle $\omega t$.

\section{REFERENCES:}

[1]. Alireza S. and Sahai V. Heat transfer in developing magnetohydrodynamic poiseuille flow and variable Transport properties, International Journal of Heat Mass Transfer, vol.33 (8), pp.1711-1720(1990).

[2]. Chamkha A.J. Flow of two-immiscible fluids in porous and non-porous channels, ASME Journal 


\section{Available online at $w w w . i j r a t . o r g$}

of Fluids Engineering, vol.122, pp.117124(2000).

[3]. Chamkha A.J. Unsteady MHD convective heat and mass transfer past a semi-infinite vertical permeable moving plate with heat absorption, International Journal of Engineering Science, vol.42, pp.217-230(2004).

[4]. Chan C.K. Finite element formulation and solution of nonlinear heat transfer, Journal of Nuclear Engineering and Design, vol.51, p.253 (1979).

[5]. Chao J., Mikic B.B. and Todreas N.E. Radiation streaming in power reactors: proceedings of the special session, American Nuclear Society (ANS) Winter Meeting, Washington, D.C. Nuclear Technology, 42, 22(1979).

[6]. Das B.K., Guria M. and Jana R.N. Unsteady Couette flow in a rotating system, Meccanica, vol.43, p.517|(2008).

[7]. Debnath L. and Basu U. Unsteady slip flow in an electrically conducting two-phase fluid under transverse magnetic fields, Nuovo Cimento, vol.28B,pp.349-362(1975).

[8]. Dunn P.F. Single-phase and two-phase magneto hydro dynamic pipe flow, International Journal of Heat Mass Transfer, vol.23, p.373 (1980).

[9]. Ganapathy R. A note on oscillatory Couette flow in a rotating system. ASME, Journal of Applied Mechanics, vol.61, p.208 (1994).

[10]. Gherson P. and Lykoudis P.S. Local measurements in two-phase liquid-metal magneto-fluid mechanic flow, Journal of Fluid Mechanics, vol.147, pp.81-104(1984).

[11]. Ghosh S.K. Unsteady hydromagnetic flow in a rotating channel with oscillating pressure gradient, Journal of the Physical Society of Japan, vol.62, p.3893 (1993).

[12]. Ghosh S.K. and Pop I. Hall effects on unsteady hydro magnetic flow in a rotating system with oscillatory pressure gradient, International Journal of Applied Mechanics and Engineering, vol.8, p.43 (2003).

[13]. Greenspan H.P. and Howard L.N. On a time dependent motion of a rotating fluid, Journal of Fluid Mechanics, vol.17, p.385 (1963).

[14]. Guria M. and Jana R.N. Hydro magnetic flow in the Ekman layer on an oscillating porous plate. Magneto hydro dynamics, vol.43, pp.311(2007).

[15]. Haim H.B., Jianzhong Z., Shizhi Q. and Yu X. A magneto-hydrodynamically controlled fluidic network, Sensors and Actuators B, vol.88 (2), pp.205-216(2003).

[16]. Hayat T. and Hutter K. Rotating flow of a second-order fluid on a porous plate, International Journal of Non-Linear Mechanics, vol.39, p.767 (2004).

[17]. Hayat T., Nadeem S. and Asghar S. Hydromagnetic Couette flow of an Oldroyd-B fluid in a rotating system , International Journal of Engineering Science, vol.42, p.65 (2004).

[18]. Hayat T., Nadeem S., Asghar S. and Siddiqui A.M. Fluctuating flow of a third-grade fluid on a porous plate in a rotating medium, International Journal of Non-Linear Mechanics, vol.36, p.901 (2001).

[19]. Heavy J.V. and Young H.T. Oscillating twophase channel flows, Z. Angew. Math. Phys.,vol.21, p.454 (1970).

[20]. Holton J. R. The influence of viscous boundary layers on transient motions in a stratified rotating fluid, International Journal of Atmospheric Science, vol.22, p.402 (1965).

[21]. Hussameddine S.K.,Martin J.M. and Sang W.J. Analytical prediction of flow field in magneto hydro dynamic based micro fluidic devices, Journal of Fluids Engineering, vol.130(9), p.6(2008)

[22]. Jana R.N. and Datta N. Couette flow and heat transfer in a rotating system Acta Mechanica,vol.26, p.301 (1977).

[23]. Lielausis O. Liquid metal magneto hydrodynamics, Atomic Energy Review, vol.13, p.527 (1975).

[24]. Linga Raju T. and Murty P.S.R. Hydro magnetic two-phase flow and heat transfer through two parallel plates in a rotating system Journal of Indian Academy of Mathematics, Indore, India, vol.28 (2), pp.343-360(2006).

[25]. Linga Raju T. and Sreedhar S. Unsteady twofluid flow and heat transfer of conducting fluids in channels under transverse magnetic field International Journal of Applied Mechanics and Engineering, vol.14(4), pp.1093-1114(2009).

[26]. Lohrasbi J. and Sahai V. Magnetohydrodynamic heat transfer in two-phase flow between parallel plates Applied Scientific Research, vol.45, pp.53-66(1989).

[27]. Malashetty M.S. and Leela V. Magnetohydrodynamic heat transfer in two phase flow International Journal of Engineering Science, vol.30, pp.371-377(1992).

[28]. Malashetty M.S. and Umavathi J.C. Two phase magneto hydrodynamic flow and heat transfer in an inclined channel International Journal of Multiphase Flow, vol.23 (3), pp.545560(1997).

[29]. Mazumder B.S. An exact solution of oscillatory Couette flow in a rotating system ASME Journal of Applied Mechanics, vol.58, p.1104 (1991).

[30]. Michiyoshi Funakawa Kuramoto C., Akita Y. and Takahashi O. Instead of the helium-lithium annular mist flow at high temperature, an airmercury stratified flow in a horizontal rectangular duct in a vertical magnetic field International Journal of Multiphase Flow,vol.3, p.445(1977) 
[31]. Mukherjee S. and Debnath L. On unsteady rotating boundary layer flows between two porous plates ZAMM, vol.57, p.188 (1977).

[32]. Packham B.A. and Shail R. Stratified laminar flow of two immiscible fluids. Proceedings of Cambridge Philosophical Society, vol.69, pp.443-448(1971).

[33]. Rahman M.M. and Sattar M.A. MHD free convection and mass transfer flow with oscillating plate velocity and constant heat source in a rotating frame of reference, The Dhaka University Journal of Science, vol.47, p.63(1999).

[34]. Ramadan H.M. and Chamkha A.J. Two-phase free convection flow over an infinite permeable inclined plate with non-uniform particle-phase density, International Journal of Engineering Science, vol.37, p.1351 (1999).

[35]. Serizawa A., Ida T., Takahashi O. and Michiyoshi I.MHD effect on Nak-nitrogen twophase flow and heat transfer in a vertical round tube International Journal Multi-Phase Flow,vol.16 (5), p.761 (1990).

[36]. Seth G.S. and Ansari M.S. Magneto hydro dynamic convective flow in a rotating channel with Hall effects International Journal of Theoretical and Applied Mechanics, vol.4, p.205 (2009).

[37]. Seth G.S. and Jana R.N.Unsteady hydro magnetic flow in a rotating channel with oscillating pressure gradient Acta Mechanica, vol.37, p.29 (1980).

[38]. Seth G.S., Jana R.N. and Maiti M.K. Unsteady hydro magnetic Couette flow in a rotating system International Journal of Engineering Science, vol.20, p.989 (1982).

[39]. Shail R. On laminar tow-phase flow in magneto hydro dynamics International Journal of Engineering Science, vol.11, p.1103 (1973).

[40]. Siegmann W.L. The spin-down of rotating stratified fluids Journal of Fluid Mechanics,vol.47, p.689 (1971).

[41]. Singh K.D. An oscillatory ydromagnetic Couette flow in a rotating system ZAMM, vol.80, p.429 (2000).

[42]. Tsuyoshi I. and Shu-Ichiro I. Two-fluid magneto hydrodynamic simulation of converging Hi flows in the Interstellar medium The Astrophysical Journal, vol.687(1), pp.303-310(2008).

[43]. Umavathi J.C., Abdul Mateen, Chamkha A.J. and Al- Mudhaf A. Oscillatory Hartmann twofluid flow and heat transfer in a horizontal channel International Journal of Applied Mechanics and Engineering, vol.11 (1), pp.155178(2006).

[44]. Vidyanidhi V. Secondary flow of a conducting liquid in a rotating channel Journal of Mathematical and Physical Sciences, vol.3, p.193 (1969).
[45]. Walin G. Some aspects of time dependent motion of a stratified rotating fluid Journal of Fluid Mechanics, vol.36, p.289 (969).

[46]. Weston M.C., Gerner M.D. and Fritsch I. Magnetic fields for fluid motion Analytical Chemistry, vol.82 (9), pp.3411-3418(2010).

[47]. Yi M., Qian S. and Bau H. A magneto hydro dynamic chaotic stirrer Journal of Fluid Mechanics, vol.468, pp.153-177(2002) 